\title{
Introduction au numéro spécial sur la ré-imagination de l'élément « $M$ » des STEM : démarches mathématiques pour des communautés innovantes, résilientes et riches en culture
}

\author{
Cynthia Nicol (i) - Kathleen Nolan (D) \\ Florence Glanfield • Krista Francis $\mathbb{D}$ \\ Published online: 11 June 2020 \\ (C) Ontario Institute for Studies in Education (OISE) 2020
}

En 2018, un rapport du Groupe d'experts intergouvernemental sur l'évolution du climat (GIEC) des Nations Unies annonçait que la température moyenne mondiale était de $1^{\circ} \mathrm{C}$ plus élevée comparativement au niveau préindustriel (GIEC, 2018). Le rapport attribuait ce réchauffement à des causes humaines, et affirmait que l'influence humaine était « devenue le principal agent de changement de la planète » (IPCC/GIEC, 2018, p. 53). Des preuves de ce changement se sont fait sentir dans le monde entier : conditions météorologiques mortelles aux États-Unis et en Asie du Sud-Est, sécheresse extrême en Afrique du Sud et feux de forêt boréaux inusités dans l'Arctique, tous en 2018. Les appels annonçant les crises écologiques de la Terre sont désormais mondiaux et difficiles à ignorer. Comment en est-on arrivé à une telle situation? Certains soutiennent que la crise écologique découle d'une vision particulière du monde, d'une manière d'être qui privilégie l'individualisme, le consumérisme comme synonyme de progrès et l'utilisation des ressources énergétiques non renouvelables comme des droits essentiels de l'humanité (Barwell, 2018; Bowers, 2017). Reconnaître la crise conduit à s'interroger sur le rôle que l'éducation peut et doit jouer pour répondre aux défis écologiques. Plus précisément, nous nous demandons comment l'enseignement des mathématiques, la formation des enseignants de mathématiques et l'enseignement des STEM (sciences, technologie, ingénierie et mathématiques) sont susceptibles de contribuer à la compréhension de la question et à y donner des réponses. Comment les mathématiques et l'enseignement des STEM peuvent-ils être repensés pour contribuer à des pratiques plus durables, ainsi qu'à des communautés plus innovantes, résilientes et

\author{
C. Nicol $(\bowtie)$ \\ University of British Columbia, 2125 Main Mall, Vancouver B.C. V6T 1Z4, Canada \\ e-mail: cynthia.nicol@ubc.ca \\ K. Nolan \\ Faculty of Education, University of Regina, 3737 Wascana Parkway, Regina, Saskatchewan S4S 0A2, Canada \\ e-mail: kathy.nolan@uregina.ca \\ F. Glanfield \\ Vice-Provost Indigenous (Programming and Research), University of Alberta, 2-40 South Academic Building, Edmonton, AB \\ T6G 2G7, Canada \\ e-mail: glanfiel@ualberta.ca \\ K. Francis \\ Werklund School of Education, University of Calgary, 2500 University Drive N.W, Calgary, Alberta T2N 1N4, Canada \\ e-mail: kfrancis@ucalgary.ca
}


culturellement plus riches? Ce numéro spécial de la RCESMT explore ces questions dans des contextes nationaux et internationaux, dans le but de repenser le rôle des STEM et de l'enseignement des STEM pour envisager d'autres façons de relier les personnes, les mathématiques et les communautés.

L'accent mis sur les STEM et l'enseignement des STEM vient de plusieurs sources, y compris les tests comparatifs internationaux, la pénurie de travailleurs en STEM et le besoin d'innovation technologique sur des marchés commerciaux internationaux toujours compétitifs (Atkinson et Mayo, 2010; European Parliament, 2015; Gonzalez et Kuenzi, 2012; Government of Canada, 2018; Avis scientifiques de la Société royale du Canada, 2014). Bon nombre de ces préoccupations s'inscrivent dans un contexte socioéconomique mondialisé hautement concurrentiel. Les rapports gouvernementaux et politiques du monde entier soutiennent que, à l'ère postindustrielle, l'économie mondiale, en mutation continue, requiert une main-d'œuvre formée en STEM (European Parliament, 2015; Government of Canada, 2018; Avis scientifiques de la Société royale du Canada, 2014). Aux États-Unis, par exemple, Committee on STEM Education of the National Science and Technology Council (2018) estiment que les STEM sont essentiels pour que les États-Unis soient « le leader mondial de l'alphabétisation, de l'innovation et de l'emploi en STEM» (p. V) et qu'ils soient « en mesure de rivaliser à l'échelle mondiale en termes de talents scientifiques et technologiques »(p. 1). De même, au Royaume-Uni, le livre blanc sur la stratégie industrielle (Gouvernement de SM britannique, 2017) a examiné des approches visant à accroître la productivité économique et les revenus des personnes, soulignant qu'une amélioration de l'enseignement des STEM serait une intervention clé. Quant à la Chine, elle reconnaît que, même avec sa population nombreuse, elle « fait face à une grave pénurie de talents innovants » dans les disciplines STEM (Wang et al., 2018, p. para. 3). À l'instar d'autres pays, le Bureau du scientifique en chef australien souligne la nécessité d'une maind'œuvre spécialisée ayant des compétences en STEM, car l'avenir de l'Australie « est un avenir qui comprend les STEM » (Office of the Chief Scientist, 2016, p. iii).

Ce numéro spécial aborde les STEM et l'enseignement des STEM d'un point de vue différent. Allant audelà, mais aussi en tenant compte, des questions de curriculum visant à construire une économie forte, ce numéro se penche sur le rôle des mathématiques (l'élément « $\mathrm{M}$ » des STEM), ainsi que sur le développement durable, l'équité, l'appartenance autochtone et les communautés rurales mal desservies en matière d'enseignement des STEM. L'enseignement des STEM au Canada offre une vision différente de l'apprentissage des STEM pour les diplômés, selon le rapport Canada 2067 (Parkin, 2018). Contrairement aux rapports nationaux d'autres pays, ce document indique que « l'objectif de Canada 2067 n'est pas d'orienter un plus grand nombre d'étudiants vers des domaines d'études et des carrières en STEM » (Parkin, 2018, p. 6). Plutôt, le rapport souligne le besoin de compétences en STEM chez tous les étudiants, dans toutes les carrières et pour tous les citoyens, et se concentre sur l'enseignement des STEM en tant que contexte d'apprentissage de la pensée critique, de la résolution de problèmes et de la compréhension interculturelle. Canada 2067 considère ces compétences comme essentielles pour intéresser les étudiants à des problèmes mondiaux complexes tels que l'accès à une éducation de qualité pour tous, l'élimination de la faim dans le monde et la lutte contre les changements climatiques. En effet, le Cadre d'apprentissage des STEM de Canada 2067 reconnaît la nécessité d'un apprentissage professionnel et d'un soutien continus pour accroitre la confiance et l'assurance des enseignants afin qu'ils puissent incorporer d'autres visions du monde et d'autres cultures, en particulier les modes de connaissance et les perspectives autochtones, dans l'enseignement et l'apprentissage des STEM (Parkin, 2018). De ce point de vue, l'enseignement des STEM ne vise pas à fournir davantage de contenus mathématiques et scientifiques aux élèves; il vise plutôt à une refonte profonde des procédés pour qu'ils puissent favoriser la participation des élèves, lesquels seront ainsi en mesure de résoudre certains des problèmes urgents de notre époque (Davis et al., 2019).

Dans leur critique de l'enseignement des STEM, Wolfmeyer et al. (2017) d'une part, et Kumashiro (2018) et Zeidler (2016) d'autre part, plaident pour une éducation allant au-delà de la maitrise des disciplines STEM. Arguant contre une vision de l'éducation centrée sur les STEM, Zeidler (2016) qualifie de déficitaire un modèle d'enseignement des STEM axé sur une éducation qui néglige de prendre en 
considération les perspectives mondiales contextualisant les disciplines. Zeidler (2016) plaide plutôt pour un type d'enseignement des STEM dans lequel :

[...] il est fondamental que tout élève puisse encadrer n'importe quel sujet issu des STEM dans un contexte personnel, réfléchi et significatif, afin de permettre une recherche ouverte, et un discours et un raisonnement fondés sur des preuves (p. 12).

Kumashiro (2018) et Wolfmeyer et al. (2017) nous invitent à critiquer les mathématiques et les sciences de l'enseignement des STEM qui sont présentées dans le cadre d'appels visant à la revitalisation économique et la compétitivité mondiale. Kumashiro (2018) rappelle que les mathématiques «ne jouent pas moins un rôle colonisateur que toute autre discipline » (p. 5), et il ajoute que les mathématiques peuvent perpétuer des injustices même si elles sont également un outil permettant de traiter des problèmes sociaux, écologiques et culturels complexes. En soi, l'enseignement des STEM peut en fait constituer un lieu où l'enseignement des mathématiques et des sciences participe à un dialogue critique entre disciplines, qui transcende les frontières de curriculum et les questions analysées. Cela signifie que les dialogues entre l'enseignement des STEM, l'enseignement des mathématiques et l'enseignement critique sont essentiels pour renforcer la démocratie, et qu'ils peuvent, comme le suggère Kumashiro (2018), « perturber les enseignements eux-mêmes » (p. 6).

En perturbant l'éducation elle-même, Bowers (2017) soutient que nous pourrions commencer par travailler « dans un paradigme qui ne colonise pas les autres cultures » (p. 54). L'éducation, cependant, est une pratique colonisatrice. La Commission de vérité et réconciliation du Canada (CVR, 2015a) rend compte des relations passées, présentes et futures entre les peuples autochtones et non autochtones, entre les colonisateurs et les colonisés, entre une éducation conçue pour isoler et assimiler de force les enfants autochtones dans le Canada traditionnel et une éducation ancrée dans les liens avec le territoire. L'histoire des pensionnats indiens au Canada est complexe et longue, la dernière école ayant fermé ses portes en Saskatchewan en 1996. Ces écoles, financées par le gouvernement canadien et dirigées par les églises du Canada, retiraient des enfants de leur famille et de leur communauté, dans le cadre d'un processus de domination culturelle entraînant une perte intergénérationnelle de la langue, des traditions et des pratiques culturelles. Les survivants de ces écoles ont déclaré avoir été la cible de graves troubles émotionnels et de violences traumatiques de la part des administrateurs scolaires et des enseignants (Truth and Reconciliation Commission of Canada (TRC), 2015a). Il est inquiétant de constater qu'environ 6000 enfants sont morts dans des pensionnats indiens, « sous prétexte de recevoir une éducation » (Talaga, 2018, p. 65). Les effets des pensionnats indiens au Canada sont profonds. "C'est l'éducation qui nous a mis dans ce pétrin », déclare le juge Murray Sinclair, alors président de la CVR, " mais l'éducation est aussi la clé de la réconciliation » (Watters, 2015).

La réconciliation, ou ce que le chercheur autochtone Styres (2017) appelle " la guérison », est un processus complexe. D'une part, la réconciliation invite les Canadiens non autochtones, les colons et les nouveaux arrivants à faire partie de l'histoire du Canada, de pair avec les peuples autochtones, à comprendre les processus de colonisation et l'élaboration des politiques historiques, et à transcender les sentiments de déni et de culpabilité pour aller vers des pratiques décolonisantes essentielles, qui incluent la renégociation des identités et l'analyse des préjugés implicites (Cote-Meek, 2014; Regan, 2010). D'autre part, la réconciliation incite les peuples autochtones à participer à la bonification des rapports avec la cérémonie, la langue et la culture, vers une «(re)-centralisation des principes, des philosophies et des ontologies de la Terre » (Styres, 2017, p. 74), en vue de la souveraineté et de l'autogouvernement (Tuck et Yang, 2018).

Pour le spécialiste des questions autochtones Alfred (2009), cependant, le processus de réconciliation pour les Canadiens est moralement défectueux, car il repose sur « une fausse notion d'équivalence morale » (p. 51) entre les peuples autochtones et non autochtones. Alfred (2009) soutient que la réconciliation exige que les peuples autochtones s'intègrent dans l'héritage colonial sans bénéficier de programmes susceptibles de faire une différence substantielle dans leur vie, alors que pour les non-Autochtones, la réconciliation n'est guère menaçante, puisqu'elle ne nécessite qu'une « simple déclaration de regret, accompagnée d'une intention de cesser les formes de racisme les plus ouvertement déclarées » (Alfred, 2009, p. 152). Selon Alfred (2009), 
l'obstacle à la réconciliation repose principalement sur la majorité non autochtone « et son déni délibéré de la réalité historique [qui] entrave toute possibilité de discussion significative sur une véritable réconciliation » (p. 152). Le rapport et les appels à l'action du CRT du Canada répondent aux préoccupations d'Alfred (2009) en ouvrant des possibilités de discussion et d'action utiles pour la réconciliation.

Les établissements d'enseignement du Canada répondent pour leur part aux recommandations de la CVR et aux 94 appels à l'action (CVR, 2015b) pour la réconciliation. De nombreuses universités à travers le Canada exigent que tous les étudiants de didactique suivent un cours d'éducation autochtone, et certaines universités exigent un tel cours pour tous les étudiants de premier cycle. Le curriculum provincial des écoles élémentaires et secondaires, par exemple celui de la Colombie-Britannique et celui de l'Ontario, requiert l'intégration des savoirs et des façons d'être autochtones dans le curriculum de toutes les matières. Pourtant, bien que ces exigences éducatives soient en place dans de nombreuses institutions, la décentralisation des structures sociales coloniales et des relations de pouvoir continue d'être difficile. En d'autres termes, le fait que l'éducation soit liée à des institutions colonisatrices telles que les universités et les écoles rend tout effort de réconciliation difficile et complexe. Cela est particulièrement vrai dans le domaine de l'enseignement des mathématiques, où les mathématiques sont souvent considérées comme une discipline acculturelle décontextualisée. Bishop (1990) accuse en fait les mathématiques d'être « l'une des armes les plus puissantes de l'imposition de la culture occidentale » (p. 51), une « arme secrète de l'impérialisme culturel » (p. 51).

Ce numéro spécial présente une collection d'études et d'enquêtes qui abordent avec respect la construction et le recentrage des relations avec les communautés, vers une décolonisation des pratiques éducatives en particulier dans l'élément « $\mathrm{M}$ » des STEM, pour reconcevoir un engagement plus holistique avec les environnements humains et naturels. Les articles de ce numéro s'inscrivent dans des perspectives qui font partie et qui vont au-delà des domaines STEM, pour analyser individuellement et collectivement les processus de décolonisation des pratiques de l'enseignement des mathématiques, pour imaginer une nouvelle vison de l'élément « $\mathrm{M}$ » des STEM et pour reconcevoir les STEM de façon à ce que les jeunes, les enseignants, les aînés et les communautés puissent participer à la création et au maintien de communautés innovantes, résilientes et culturellement riches. Les articles fournissent des exemples d'éveil, ce que Shaughnessy (2013) appelle l'élément « M silencieux des STEM » (p. 324), à travers des projets convaincants situés dans des contextes qui ne sont généralement pas pris en compte dans les programmes de recherche en STEM et en enseignement des STEM. Bien qu'il existe de nombreuses intersections et des intérêts qui se chevauchent parmi les six articles de ce numéro spécial, tous se penchent, de manière parfois désordonnée, complexe ou tendue, sur qu'implique une nouvelle conception de l'élément « $\mathrm{M}$ » des STEM.

Nous commençons avec Nicol et al. (2020) qui analysent leurs propres expériences en formation des enseignants de mathématiques pour repenser l'élément « $\mathrm{M}$ » des STEM. Dans leur article L'apprentissage professionnel des enseignants et le récit situé sur le plan géographique, pour décoloniser l'enseignement des mathématiques au sein de structures coloniales, les auteurs explorent leurs diverses expériences collectives pour accroître leur sensibilisation aux pratiques de colonisation. S'appuyant sur la méthodologie narrative de Clandinin ( « vivre, raconter, raconter de nouveau et revivre »), l'article examine les façons d'être et d'interagir dans des contextes variés, y compris un jardin d'apprentissage pour les futurs enseignants, un cours en classe sur l'éducation culturellement adaptée destiné aux enseignants, et des leçons de robotique destinées aux jeunes Autochtones dans une communauté rurale éloignée. Les tensions sont partagées et de nouvelles possibilités sont explorées alors que Nicol et al. proposent un « voyage collaboratif en invitant d'autres chercheurs à revivre l'apprentissage des mathématiques ensemble, pour apaiser et guérir les cicatrices infligées à la terre et à nous-mêmes, un voyage où des visions irrégulières du monde entrent en collision ». Comme pour l'article de Khan (2020) les auteurs explorent l'élément « M » des STEM pour « ré-imaginer les liens avec les mathématiques, les lieux, les communautés et l'éducation ».

Passant de la formation des enseignants de mathématiques à l'enseignement des mathématiques au premier cycle, notre deuxième article met en évidence les expériences de mathématiciens qui cherchent à améliorer l'expérience des étudiants de premier cycle non spécialisés en STEM. Dans Ré-imaginer de nouvelles tâches mathématiques authentiques pour les étudiants non spécialisés en STEM, Leung et al. 
(2020) décrivent chacun une tâche authentique complexe/désordonnée ayant mobilisé l'intérêt et les expériences antérieures de leurs élèves. Cet article traite de tâches qui comprennent : la représentation graphique des taux d'incarcération pour comprendre les relations linéaires, quadratiques et exponentielles ; le comptage des carreaux dans des couvertures étoilées de tailles croissantes pour comprendre les séquences quadratiques ; et l'extrapolation, par les élèves, de la datation au radiocarbone pour leur permettre de comprendre la décroissance exponentielle des restes d'êtres humains préhistoriques mangeurs de poissons. Les auteurs soulignent le défi d'intégrer des tâches authentiques complexes/désordonnées dans des cours universitaires de mathématiques, et la nécessité de structurer l'apprentissage des élèves non spécialisés en STEM. Sachant que ce type d'enseignement peut être difficile pour les étudiants et les enseignants, les auteurs insistent sur l'importance des communautés professionnelles de soutien pour partager, et remettre en question, les idées. Ils concluent en citant la nécessité d'une « innovation continue dans les cours de mathématiques à l'université » afin que les étudiants spécialisés en STEM et ceux qui ne le sont pas établissent des relations positives avec les mathématiques.

Dans Perspectives « situées » sur la création de tâches mathématiques pour la paix et la durabilité, Yaro et al. (2020) analysent des tâches mathématiques authentiques dans une perspective internationale. Dans ce troisième article du numéro spécial, les auteurs se penchent sur leurs expériences d'enseignement au Canada et ailleurs, soulignant les tensions qui existent entre la colonisation mondialisée et l'attention aux contextes locaux si on veut créer des tâches mathématiques authentiques et significatives. Yaro et al. explorent les façons dont la paix, la durabilité et les problèmes locaux peuvent éclairer l'enseignement traditionnel des sciences et des mathématiques. Parmi les défis à affronter pour la mise en œuvre d'un tel programme, les auteurs indiquent la nature conservatrice des manuels scolaires, le discours dominant de la technoscientificité, la colonisation et les contraintes de temps. Les auteurs se servent d'un exemple d'échange d'argent au Ghana comme tâche authentique localisée et contextualisée pour repenser, dans une perspective interdisciplinaire, l'élément « $\mathrm{M}$ » des STEM.

Alors que les articles de Yaro et al. (2020) et de Nicol et al. (2020) soulignent la nécessité d'un enseignement des mathématiques favorisant une sensibilisation aux pratiques de colonisation dans le cadre de problèmes contextualisés, les deux articles suivants abordent les perspectives écologiques à travers une exploration intentionnelle des différents sens que pourrait prendre l'élément « $\mathrm{M} »$ des STEM. Dans Après l'élément « $M$ » de «STEM» : vers un épanouissement multi-espèces, Steven Khan (2020) se propose de ré-imaginer cet « M» en faisant appel aux idées de la philosophe et romancière Sylvia Wynter, en particulier sa critique culturelle de de ce que signifie être « humain ». Khan joue avec différents sens possibles de « $\mathrm{M} »(\mathrm{M}$ pour « Man » (Humain), pour « après Humain », pour « multi-espèces », pour « musique »). S'appuyant sur le projet de Wynter visant à réunir l'Humain et l'écologique, Khan explore habilement « l'après-M des STEM » pour redéfinir l'être humain vers l'épanouissement multi-espèces et la liberté. Cet article souligne la façon dont les mathématiques et l'enseignement des STEM ont des structures de connaissances qui sont à la fois créées et perpétuées dans certains types de logique, comme la logique des plantations, l'économie et le colonialisme. Contestant les discours qui font appel aux STEM et à l'enseignement des STEM pour résoudre les problèmes actuels du monde, Khan fait valoir la nécessité d'actions, y compris des conceptions alternatives de la temporalité, qui permettent plutôt « l'épanouissement non seulement pour nous-mêmes en tant qu'êtres humains, mais aussi pour tous les liens auxquels nous participons ». Khan se fonde sur ses propres expériences pédagogiques pour fournir des exemples de façons dont les mathématiques et l'enseignement des mathématiques peuvent être des lieux de réinvention et de reconnexion avec nos relations et avec la terre, des lieux où la communauté, les êtres humains, les non-humains et l'environnement coexistent et s'épanouissent.

De manière similaire, Glanfield et al. (2020) dans leur article Paysages vivants, architectures, topographie et « algo-rythmes », ré-imaginent les STEM dans des perspectives autochtones et écologiques. L'article analyse les formes culturelles de connaissance, en particulier celles qui sont profondément liées à l'environnement naturel, pour explorer d'autres possibilités des STEM enracinées dans des perspectives autochtones et écologiques. Les auteurs invitent le lecteur à jouer avec les idées de paysages vivants, de topographie, d'architecture et « d'algo-rythmes », de concert avec des activités architecturales des peuples 
autochtones, pour remettre en question certaines significations acceptées des STEM et imaginer les STEM à travers des formes de savoir profondément ancrées dans l'environnement naturel.

L'équipe de Dawn Wiseman et al. (2020) poursuit l'exploration des significations alternatives des STEM. Elles rassemblent les expériences visant «à créer des occasions d'apprentissage où les savoirs autochtones et occidentaux, de même que les différentes façons d'être et de faire, pourraient se côtoyer ». Dans un article intitulé Artéfacts authentiques : l'enseignement et l'apprentissage (des STEM) qui émergent des communautés et peuvent y contribuer, l'équipe relate le travail réalisé dans le cadre de trois projets, dans trois communautés différentes, «pour analyser comment les STEM peuvent émerger des communautés, et aussi comment elles peuvent contribuer à ces communautés ». Bien que les auteures expriment leur réticence à utiliser le terme de STEM pour décrire le travail qu'elles font dans leurs divers contextes et communautés, elles proposent une façon de voir l'élément « $M$ » des STEM par le biais de projets et de sites où les mathématiques ont émergé de manière plus organique, et où les STEM sont vécues « comme un artefact vivant et authentique d'enseignement et d'apprentissage, au lieu d'un ensemble de structures disciplinaires imposées à l'enseignement et à l'apprentissage ».

Chacun des articles de ce numéro spécial offre aux lecteurs une ré-imagination innovante et critique du rôle des mathématiques dans les domaines des STEM et dans leur enseignement. Deux commentaires suivent les articles : un par Yasmine Abtahi, de l'Université des sciences appliquée de la Norvège occidentale, et l'autre par Michael Marker et Shirley Anne Hardman, de l'Université de la ColombieBritannique. Abtahi (2020) apporte une expérience de travail dans les domaines de l'enseignement des mathématiques autochtones et culturellement adaptés dans des contextes internationaux. Marker and Hardman (2020) offrent des perspectives extérieures au domaine de la recherche en enseignement des mathématiques, en s'inspirant de leurs propres expériences d'apprentissage des mathématiques et de leurs expériences actuelles en tant que chercheurs et administrateurs autochtones. Nous laissons aux lecteurs certaines questions à prendre en compte lors de leur lecture des articles de ce numéro, en espérant qu'elles resteront présentes à l'esprit et les pousseront à y revenir :

- Comment vivez-vous l'élément « $\mathrm{M} »$ des STEM ?

- Doit-on obligatoirement ré-imaginer les mathématiques des STEM ? À qui s'adresse cette obligation et pour qui devrait-on le faire?

- Quel est le rôle que les communautés autochtones jouent ou peuvent jouer pour repenser les voies d'accès aux STEM et à l'enseignement des STEM ?

- Comment les mathématiques peuvent-elles participer à cette ré-imagination des STEM et de l'enseignement des STEM, ainsi qu'à la décolonisation des pratiques éducatives ?

- Comment la question de repenser l'élément « $\mathrm{M}$ » des STEM s'applique-t-elle, ou pourrait-elle s'appliquer, à vous?

Au nom de tous les auteurs, l'auteur correspondant déclare qu'il n'y a aucun conflit d'intérêts.

\section{Références}

Abtahi, Y. (2020). The "M" in STEM as a note of caution: Resilient to what and responsive to whose culture. Canadian Journal of Science, Mathematics and Technology Education, 20(2). doi: https://doi.org/10.1007/s42330-020-00093-8.

Alfred, T. (2009). Wasáse: Indigenous pathways of action and freedom. Toronto, University of Toronto Press.

Atkinson, R., \& Mayo, M. (2010). Refueling the U.S. innovation economy: Fresh approaches to Science, Technology, Engineering and Mathematics (STEM) Education. Information Technology and Innovation Foundation. Retrieved from https://itif.org/. Accessed 8 June 2020.

Barwell, R. (2018). Some thoughts on a mathematics education for environmental sustainability. In P. Ernest (Ed.) The philosophy of mathematics education today (pp. 145-160). New York: Springer.

Bishop, A. J. (1990). Western mathematics: The secret weapon of cultural imperialism. Race and Class, 32(2), 51-65. 
Bowers, C. A. (2017). An ecojustice approach to educational reform in adult education. New Directions for Adult and Continuing Education, 2017(153), 53-64. https://doi.org/10.1002/ace.20221

Committee on STEM Education of the National Science and Technology Council (2018). Charting a course for success: America's strategy for STEM education. Retrieved from: https://www.whitehouse.gov/wp-content/uploads/2018/12 /STEM-Education-Strategic-Plan-2018.pdf. Accessed 8 June 2020.

Cote-Meek, S. (2014). Colonized classrooms: Racism, trauma and resistance in post-secondary education. Winnipeg: Fernwood Publishing.

Davis, B., Francis, K., \& Friesen, S. (2019). STEM education by design: Opening horizons of possibility. New York, NY: Routledge.

European Parliament (2015). Encouraging STEM studies for the labour market. http://www.europarl.europa.eu/studies. Accessed: 11 Mar 2019.

Glanfield, F., Thom, J., \& Ghostkeeper, E. (2020). Living landscapes, archi-text-ures, and land-guaging algo-rhythms. Canadian Journal of Science, Mathematics and Technology Education, 20(2). https://doi.org/10.1007/s42330-020-00085-8.

Gonzalez, H., \& Kuenzi, J. (2012). Science, technology, engineering, and mathematics (STEM) Education: A Primer. Washington, DC: Library of Congress, Congressional Research Service. Retrieved from https://www.fas. $\mathrm{org} / \mathrm{sgp} / \mathrm{crs} / \mathrm{misc} / \mathrm{R} 42642 . p d f$. Accessed 8 June 2020.

Government of Canada (2018). The Government of Canada and STEM. Retrieved from the Government of Canada website: https://www.ic.gc.ca/eic/site/013.nsf/eng/00014.html

Intergovernmental Panel on Climate Change [IPCC/GIEC] (2018). Special report on global warming of $1.5^{\circ} \mathrm{C}$. Retrieved from https://www.ipcc.ch/sr15/. Accessed 8 June 2020.

Khan, S. (2020). After the M in STEM: Towards mathematics for multispecies flourishing. Canadian Journal of Science, Mathematics and Technology Education, 20(2). https://doi.org/10.1007/s42330-020-00089-4.

Kumashiro, K. (2018). Why and how STEM education matters in social justice movements. The Journal of Educational Foundations, 31(1-2), 3-5.

Leung, F. S., Radzimski, V. \& Doolittle, E. (2020). Reimagining authentic mathematical tasks for non-STEM majors. Canadian Journal of Science, Mathematics and Technology Education, 20(2). https://doi.org/10.1007/s42330-020-00084-9.

Marker, M. \& Hardman, S. (2020). The math people: Unwitting agents of empire who "like to stay in their lane." Canadian Journal of Science, Mathematics and Technology Education, https://doi.org/10.1007/s42330-020-00094-7.

Nicol, C., Gerofsky, S., Nolan, K., Francis, K., \& Fritzlan, A., (2020). Teacher educator professional learning with/in place: Storying the work of decolonizing mathematics education within a colonial structure. Canadian Journal of Science, Mathematics and Technology Education, https://doi.org/10.1007/s42330-020-00080-z.

Office of the Chief Scientist (2016). Australia's STEM workforce: Science, technology, engineering and mathematics. Retrieved from https://www.chiefscientist.gov.au/2016/03/report-australias-stem-workforce/. Accessed 8 June 2020.

Parkin, A. (2018). Supporting Education Transformation in Canada. Retrieved from https://canada2067.ca/app/uploads/2018 /11/Canada-2067-Supporting-Education-Transformation-in-Canada_Final.pdf. Accessed 8 June 2020.

Regan, P. (2010). Unsettling the settler within: Indian residential schools, truth telling, and reconciliation in Canada. Vancouver: UBC Press.

Royal Society Science Policy Centre (2014). Vision for science and mathematics education. Retrieved from https://royalsociety.org/topics-policy/projects/vision/. Accessed 8 June 2020.

Shaughnessy, J. M. (2013). Mathematics in a STEM context. Mathematics Teaching in the Middle School, 18(6), 324-324. doi: https://doi.org/10.5951/mathteacmiddscho.18.6.0324

Styres, S. D. (2017). Pathways for remembering and recognizing indigenous thought in education: Philosophies of iethi'nihsténha ohwentsia'kékha (land). Toronto: University of Toronto Press.

Talaga, T. (2018). All our relations: Finding the path forward. Toronto: House of Anansi Press.

Truth and Reconciliation Commission of Canada (TRC) (2015a). Honouring the Truth, Reconciling for the Future Summary of the Final Report of the Truth and Reconciliation Commission of Canada. : www.trc.ca/. Accessed 8 June 2020.

Truth and Reconciliation Commission of Canada (TRC) (2015b). Truth and Reconciliation Commission of Canada: Calls to action. Government of Canada. Retrieved from http://nctr.ca/assets/reports/Calls_to_Action_English2.pdf. Accessed 8 June 2020.

Tuck, E., \& Yang, K. W. (2018). Introduction: Born under the rising sign of social justice. In E. Tuck \& K. W. Yang (Eds.) Toward what justice?: Describing diverse dreams of justice in education, (pp. 1-17). New York, NY: Routledge.

UK HM Government (2017). Industrial strategy: Building a Britain fit for the future. Royal United Services Institution. White paper. Retrieved from https://www.gov.uk/government/publications/industrial-strategy-building-a-britain-fit-for-the-future. Accessed 8 June 2020.

Wang, X., Xu, W., Guo, L., Wang, X., Xu, W., \& Guo, L. (2018). The status quo and ways of STEAM education promoting China's future social sustainable development. Sustainability, 10(12), 4417. https://doi.org/10.3390/su10124417.

Watters, H. (2015). Truth and reconciliation chair urges Canada to adopt UN declaration on Indigenous Peoples. CBC News, June 01, Retrieved: https://www.cbc.ca/news/politics/truth-and-reconciliation-chair-urges-canada-to-adopt-undeclaration-on-indigenous-peoples-1.3096225. Accessed 8 June 2020. 
Wiseman, D., Lunney Borden, L., Beatty, R., Jao, L., Carter, E. (2020). Whole-some artifacts: (STEM) teaching and learning emerging from and contributing to community. Canadian Journal of Science, Mathematics and Technology Education, https://doi.org/10.1007/s42330-020-00079-6.

Wolfmeyer, M., Lupinacci, J., \& Chesky, N. (2017). Critical education. Critical Education, 8(15), 37-39. https://doi. org/10.1007/978-1-137-42617-8_15

Yaro, K., Amoah, E., \& Wagner, D. (2020). Situated perspectives on creating mathematics tasks for peace and sustainability. Canadian Journal of Science, Mathematics and Technology Education, https://doi.org/10.1007/s42330-020-00083-w.

Zeidler, D. L. (2016). STEM education: A deficit framework for the twenty first century? A sociocultural socioscientific response. Cultural Studies of Science Education, 11(1), 11-26. https://doi.org/10.1007/s11422-014-9578-z

Publisher's Note Springer Nature remains neutral with regard to jurisdictional claims in published maps and institutional affiliations. 